\title{
Inhibitory Effects of Far-Infrared Irradiation Generated by Ceramic Material on Murine Melanoma Cell Growth
}

\author{
Ting-Kai Leung, ${ }^{1}$ Chin-Feng Chan, ${ }^{2}$ Ping-Shan Lai, ${ }^{3}$ Chih-Hui Yang, ${ }^{4}$ \\ Chia-Yen Hsu, ${ }^{3}$ and Yung-Sheng Lin ${ }^{2}$ \\ ${ }^{1}$ Department of Radiology, School of Medicine, Taipei Medical University and Hospital, Taipei 110, Taiwan \\ ${ }^{2}$ Department of Applied Cosmetology and Master Program of Cosmetic Science, Hungkuang University, Taichung 433, Taiwan \\ ${ }^{3}$ Department of Chemistry, National Chung Hsing University, Taichung 402, Taiwan \\ ${ }^{4}$ Department of Biological Science and Technology, I-Shou University, Kaohsiung 824, Taiwan
}

Correspondence should be addressed to Yung-Sheng Lin, linys@sunrise.hk.edu.tw

Received 20 April 2011; Revised 9 July 2011; Accepted 9 July 2011

Academic Editor: Rodica-Mariana Ion

Copyright ( $) 2012$ Ting-Kai Leung et al. This is an open access article distributed under the Creative Commons Attribution License, which permits unrestricted use, distribution, and reproduction in any medium, provided the original work is properly cited.

\begin{abstract}
The biological effects of specific wavelengths, so-called "far-infrared radiation" produced from ceramic material (cFIR), on whole organisms are not yet well understood. In this study, we investigated the biological effects of cFIR on murine melanoma cells (B16F10) at body temperature. cFIR irradiation treatment for $48 \mathrm{~h}$ resulted in an $11.8 \%$ decrease in the proliferation of melanoma cells relative to the control. Meanwhile, incubation of cells with cFIR for $48 \mathrm{~h}$ significantly resulted in $56.9 \%$ and $15.7 \%$ decreases in the intracellular heat shock protein (HSP)70 and intracellular nitric oxide (iNO) contents, respectively. Furthermore, cFIR treatment induced $6.4 \%$ and $12.3 \%$ increases in intracellular reactive oxygen species stained by 5 -(and 6)-carboxyl-2' $7^{\prime}$-dichlorodihydrofluorescein diacetate and dihydrorhodamine 123, respectively. Since malignant melanomas are known to have high HSP70 expression and iNO activity, the suppressive effects of cFIR on HSP70 and NO may warrant future interest in antitumor applications.
\end{abstract}

\section{Introduction}

Melanomas are one of the major malignant tumors of Caucasian people, with approximately 60,000 new cases of invasive melanoma being diagnosed in the USA each year. According to a WHO report, about 48,000 melanoma-related deaths occur worldwide per year. Melanocytes are normally present in the skin, and they are responsible for producing the dark pigment, melanin. Despite many years of intensive laboratory and clinical research, the greatest chance of a cure is early surgical resection of thin tumors.

Far-infrared radiation (FIR) is the major heat-transmitting radiation of sunlight at wavelengths between $3 \mu \mathrm{m}$ and $1 \mathrm{~mm}$ as defined by the International Commission on Illumination (CIE 1987). FIR, especially that at the range of $3 \sim 14 \mu \mathrm{m}$, is termed "life light" and has many biological effects. Previous studies demonstrated that FIR has a wide range of applications including increasing the microcirculation, promoting wound healing, modulating sleep, treating depression, inhibiting tumor proliferation, and processing food [1-6]. Recently, FIR use has been growing in popularity for its health-promoting properties and is an alternative remedy in Japan, China, Taiwan, and Korea. However, the mechanisms underlying these biological effects are still poorly understood. There are few reports investigating the biological effects of FIR, especially those concerned with the effect on cancer cells, such as melanomas.

This study is aimed to investigate the possible biological effects of FIR produced by ceramic material (cFIR) on murine melanoma cells using the B16-F10 cell line. We focused on the effects on cell viability, intracellular heat shock protein (HSP)70, intracellular nitric oxide (iNO), inducible nitric oxide synthase (iNOS), and reactive oxygen species (ROS). The direct suppressive effect on melanoma cells by FIR was investigated. The inhibition of HSP70 synthesis and iNO in tumor cells shows the possible utility of FIR in cancer therapy. After a literature review, we discuss the possible physiological mechanism behind these observations 


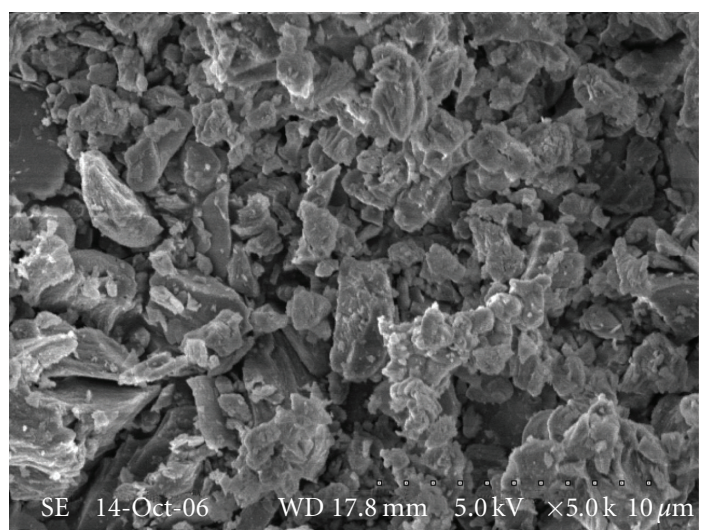

FIGURE 1: SEM picture of cFIR ceramic powder used in this study.

based on past studies detailing related biomolecular factors and future applications.

\section{Methods}

2.1. Chemicals and Reagents. B16-F10, a murine melanoma cell line (ATCC: CRL-6475), was procured from the Bioresource Collection and Research Center (Hsinchu, Taiwan). Dulbecco's modified Eagle's medium (DMEM), fetal bovine serum (FBS), sodium bicarbonate, antibiotic/antimycotic solution, and trypsin/ $0.52 \mathrm{mM}$ EDTA solution were purchased from Gibco (Grand Island, NY, USA). 3-(4,5-Dimethylthiazol-2-yl)-2,5-diphenyltetrazolium bromide (MTT), phosphate-buffered saline (PBS), dimethyl sulfoxide (DMSO), Hoechst 33342, and phenylmethylsulfonyl fluoride (PMSF) were purchased from Sigma (St. Louis, Mo, USA). The rabbit antihuman HSP70 antibody, anti- $\beta$ actin antibody, and anti-iNOS antibody were obtained from Stressgen (Victoria, BC, Canada), Abcam (Cambridge, UK), and Calbiochem (San Diego, USA), respectively. 4-Amino-5methylamino- $2^{\prime}, 7^{\prime}$-difluorofluorescein (DAF-FM) diacetate and dihydrorhodamine 123 were obtained from Invitrogen (Branford, Conn, USA). 5-(and 6)-Carboxyl-2', $7^{\prime}$-dichlorodihydrofluorescein diacetate (Carboxy-H2DCFDA) was obtained from Molecular Probes (Eugene, Ore, USA).

2.2. cFIR Ceramic Powder. As previously reported [7-9], the cFIR ceramic powder consisted of microsized particles (Figure 1) made from numerous mineral oxides, including aluminum oxide, ferric oxide, magnesium oxide, and calcium carbonate. These crushed and irregular-shaped microparticles had an average size of $4.39 \mathrm{um}$. The cFIR emissivity (the ratio of the radiation energy irradiated from a sample relative to an ideal black body, as described by Plank's law) was determined using an SR5000 infrared spectroradiometer (CI, Migdal HaEmek, Israel). The amount of FIR energy reaching the cells was $0.11 \mathrm{~J} / \mathrm{cm}^{2}$ with FIR at wavelengths between 3 and $14 \mu \mathrm{m}$. Prior to use in cell culture, $180 \mathrm{~g}$ of cFIR powder was placed in a plastic bag and sterilized with $75 \%$ ethanol and UV light.

2.3. Cell Culture. B16-F10 cells were cultured in DMEM supplemented with $10 \% \mathrm{FBS}, 1.5 \mathrm{~g} / \mathrm{L}$ sodium bicarbonate,
$4.5 \mathrm{~g} / \mathrm{L}$ glucose, $100 \mathrm{U} / \mathrm{mL}$ penicillin, $0.1 \mathrm{mg} / \mathrm{mL}$ streptomycin, and $0.25 \mu \mathrm{g} / \mathrm{mL}$ amphotericin $\mathrm{B}$ at $37^{\circ} \mathrm{C}$ with $5 \%$ $\mathrm{CO}_{2}$ in a humidified incubator. Cells were subcultured at a ratio of 1:5 every third or fifth day. To evaluate the effect of the cFIR powder, cultured cells were divided into a cFIR group and control group that received no cFIR treatment. B16-F10 cells were seeded at a density of $2 \times 10^{5}$ cells/well in 6-well plates. Following previous reports [7-9], enclosed FIR powder was uniformly distributed in a plastic bag, and the bag was inserted directly beneath the cell culture dish in the cFIR group. RAW 264.7 macrophages were used as the positive control to study the antitumor effects.

2.4. Cell Viability and Proliferation. The cell survival rate was quantified using a colorimetric MTT assay that measured mitochondrial activity in viable cells. This method was performed as previously described with slight modifications [10]. In brief, B16-F10 cells were seeded at a density of 2 $\times 10^{5}$ cells/well in 6 -well plates. Cells were then incubated for $48 \mathrm{~h}$. To evaluate the effect of the cFIR powder, cultured cells were divided into three groups: group $\mathrm{C}$ was the control without cFIR influence; group FP24 consisted of cells cultured in a normal environment for $24 \mathrm{~h}$ and then cultured with the cFIR powder for another $24 \mathrm{~h}$; group FP48 consisted of cells cultured with the cFIR powder for $48 \mathrm{~h}$. MTT was freshly prepared at $1 \mathrm{mg} / \mathrm{mL}$ in PBS, and $800 \mu \mathrm{L}$ was added to each well and incubated at $37^{\circ} \mathrm{C}$ for $4 \mathrm{~h}$. Then, $800 \mu \mathrm{L}$ of DMSO was added to each well to dissolve the MTT-formazan crystals. After incubation at $37^{\circ} \mathrm{C}$ for $10 \mathrm{~min}$, the solution was transferred to a 96-well enzyme-linked immunosorbent assay (ELISA) plate, and the absorbance was measured with a spectrophotometer at $540 \mathrm{~nm}$. The optical density (O.D.) of the control cells was considered to be $100 \%$.

2.5. HSP70. The primary polyclonal rabbit antihuman HSP70 antibody and rabbit anti- $\beta$ actin antibody were used at a 1:2000 dilution. Blots were developed using a horseradish peroxidase-conjugated goat antirabbit secondary antibody and enhanced chemiluminescence (ECL system, Amersham Biosciences). Analysis was then performed, and differences between the control and experimental groups were quantitatively determined by Winlight 32 software (Berthold Technologies).

2.6. iNO. The experimental group included 18 plates with B16-F10 cells receiving cFIR treatment for $48 \mathrm{~h}$. The control group had the same condition but without cFIR treatment. Cells were then stained with DAF-FM diacetate for fluorescence measurements. Fluorescence was analyzed by FACScan flow cytometer (Becton Dickinson, USA), and fluorescence intensity profiles and the mean fluorescence intensities of different treatments of B16-F10 cells were determined for the data analysis.

2.7. iNOS. The iNOS expression was determined by western blotting analysis. At the end of the incubation period, cells were washed with PBS, scraped with a rubber policeman, and sonicated for $2 \mathrm{~min}$ in ice-cold solution. Proteins ( $50 \mu \mathrm{g} / \mathrm{lane}$ ) were separated by electrophoresis on an $8 \%$ acrylamide gel and transferred to nitrocellulose, which was then incubated 


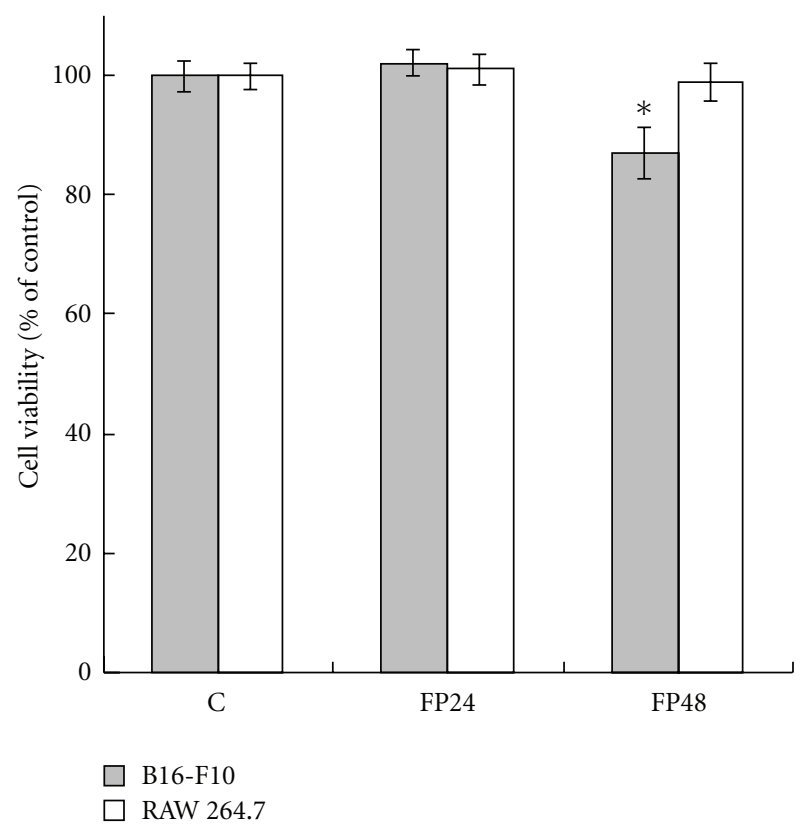

FIgURE 2: The effect of cFIR on B16-F10 and RAW 264.7 cell viability. Groups C, FP 24, and FP48 received cFIR treatment for 0,24 , and $48 \mathrm{~h}$, respectively. Values are expressed as the mean and standard deviation, and the difference between groups was tested using the Wilcoxon test.

with an anti-iNOS antibody at a 1:200 dilution. The bands corresponding to iNOS were visualized by enhanced chemiluminescence.

2.8. ROS. The intracellular ROS level of B16-F10 was measured after $48 \mathrm{~h}$ with or without cFIR treatment. Intracellular ROS were detected using Carboxy-H2DCFDA. When oxidized by ROS, Carboxy-H2DCFDA fluoresces green. After incubating cells for 30 min with $1 \mu \mathrm{M}$ of Carboxy-H2DCFDA [11], the fluorescence was detected by confocal laser scanning microscopy (SP5, Leica) with excitation and emission wavelengths of 488 and $505 \sim 560 \mathrm{~nm}$, respectively.

Intracellular ROS were also measured by flow cytometry. After $48 \mathrm{~h}$ with or without cFIR treatment, a dihydrorhodamine 123 working solution was added directly to the medium to reach $25 \mu \mathrm{M}$ and then incubated at $37^{\circ} \mathrm{C}$ for $25 \mathrm{~min}$. Cells were then washed once, resuspended in PBS, and kept on ice for immediate detection by FACScan flow cytometry [12]. Levels of dihydrorhodamine 123 fluorescence represent the values from $10^{4}$ cells based on an arbitrary scale of fluorescence intensity.

2.9. Apoptosis. A cell that is undergoing apoptosis demonstrates nuclear condensation and DNA fragmentation, which can be detected by staining with Hoechst 33342 and fluorescence microscopy. B16-F10 cells were washed with PBS and stained with Hoechst $33342(5 \mathrm{mg} / \mathrm{mL})$ for $20 \mathrm{~min}$ at room temperature to detect apoptosis. Three independent experiments were used for each group, and at least 100 cells in seven random fields were counted [13].
2.10. Statistical Analysis. All data were measured in triplicate, with experiments repeated at least three times. Data are presented as the mean \pm standard deviation. Statistical significance between the control and cFIR groups was determined using the Wilcoxon test method. A value of $P<0.05$ was considered statistically significant $(*)$, and $P<0.01$ was highly significant $(* *)$.

\section{Results}

3.1. Proliferation of Murine Melanoma Cells. Results of the cell viability assays are presented in Figure 2. For groups C (control), FP24 (cFIR irradiated for $24 \mathrm{~h}$ ), and FP48 (cFIR irradiated for $48 \mathrm{~h}$ ), cell viabilities were $100 \% \pm 2.9 \%$, $101.9 \% \pm 2.5 \%$, and $88.2 \% \pm 4.8 \%$, respectively. Compared to group $\mathrm{C}$, the rate of proliferation did not significantly change in group FP24, but significantly decreased $(11.8 \%$ lower) in group FP48. RAW 264.7 macrophages were not affected by cFIR treatment. A significant difference in the inhibitory effect on B16-F10 cell viability was found at $48 \mathrm{~h}$, and we examined HSP70, iNO, iNOS, ROS, and cell apoptosis at $48 \mathrm{~h}$ according to the cell viability results.

3.2. HSP70. After B16-F10 cells were treated with or without cFIR for a $48 \mathrm{~h}$ interval, levels of HSP70 synthesis were measured by western blotting. To normalize HSP70 contents, we evaluated the ratio of HSP70 to $\beta$-actin. Figure 3 shows that intracellular HSP70 production in the cFIR group was significantly less than that of the control group. The relative HSP70 amounts were $0.86 \pm 0.10$ in group C and $0.37 \pm$ 0.07 in group FP48. This result reveals that cFIR significantly inhibited intracellular HSP70 expression by B16-F10 cells.

3.3. iNO. Levels of NO synthesis in group FP48 subjected to a $48 \mathrm{~h}$ interval with cFIR treatment and in group C without treatment were measured by the mean fluorescence intensity. Fluorescence intensities were $14 \pm 1.4$ in group C and $11.8 \pm$ 0.5 in group FP48. Figure 4 shows that the iNO production in the cFIR group was significantly less than that of the control group. This result reveals that cFIR inhibited iNO synthesis by B16-F10 cells.

3.4. iNOS. Analysis of iNOS expression for B16-F10 cells subjected to a $48 \mathrm{~h}$ interval with or without cFIR treatment was performed by Western blotting. Figure 5 indicates that the normalized mean production of iNOS protein (iNOS/GAPDH) in group C and group FP48 are $2.85 \pm$ 1.19 and $1.33 \pm 0.75$, respectively. This result may reflect the ability of cFIR to suppress iNOS expression by B16-F10 cells.

3.5. ROS. Figure 6 shows the staining of intracellular ROS by confocal laser scanning microscopy. Green spots in the images are stained ROS. Levels of intracellular ROS for the group subjected to a 48-h interval with cFIR treatment (Figure 6(a) right image) exhibited an increased amount compared to the control group (Figure 6(a) left image). The normalized ROS level (average intracellular/extracellular fluorescence intensity) indicated that there was a $6.0 \%$ increase in the cFIR group $(1.07 \pm 0.04)$ compared to the control group (1.13 \pm 0.05$)$ as shown in Figure 6(b). 


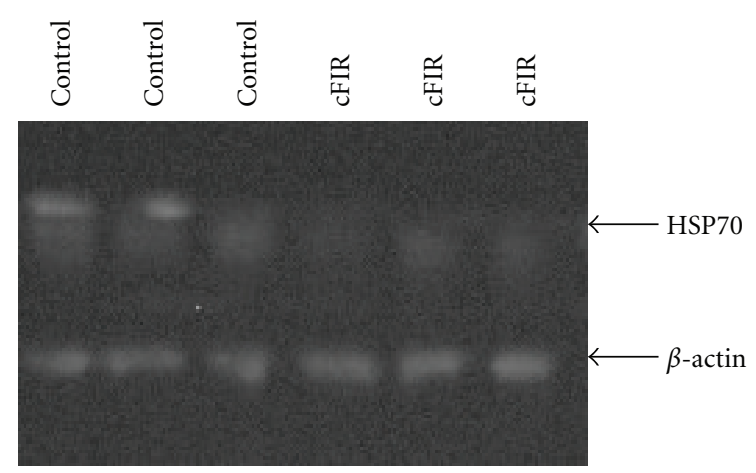

(a)

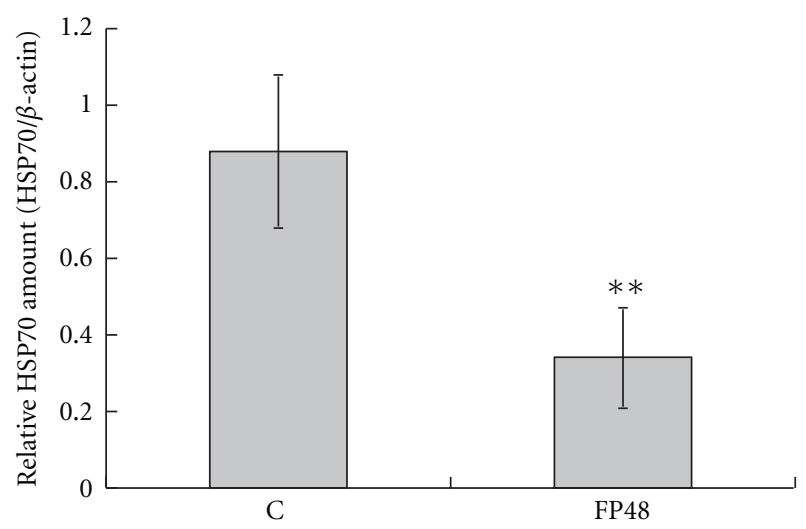

(b)

Figure 3: Comparison of intracellular HSP70 levels between the control (group C) and cFIR (group FP48) groups. (a) Western blotting gel. (b) The mean and standard deviation of the normalized HSP70 amount (Wilcoxon test).

Figure 7 shows another ROS result by flow cytometry. The mean fluorescence intensities of group $\mathrm{C}$ and group FP48 were $127.1 \pm 14.1$ and $142.7 \pm 18.0(n=12)$, respectively. Therefore, the intracellular fluorescence intensity of group FP48 showed a $12.3 \%$ increase compared to group C. This result is consistent with the finding of confocal laser scanning microscopy (Figure 6).

3.6. Apoptosis. Compared to group C, inhibition of cell proliferation was observed in group FP48 by Hoechst 33342 staining (Figure 8). Compared to group C, cell proliferation was inhibited by $13.2 \% \pm 0.8 \%$ in group FP 48 . This result is consistent with the result of the MTT assay in Figure 2. However, only about $1.1 \% \pm 0.1 \%$ cells with apoptotic changes were observed in group FP48 (arrow in Figure 8(b)). The result indicates that inhibition of cell proliferation in group FP48 was perhaps not through inducing cell apoptosis but by interfering with the cell cycle such as cell growth arrest.

\section{Discussion}

In this study, we observed that the growth of B16-F10 cells was inhibited after irradiation with cFIR for $48 \mathrm{~h}$ (Figure 2) compared to the control group. A previous in vitro study [14] revealed that FIR with a heat source (hFIR) inhibited the

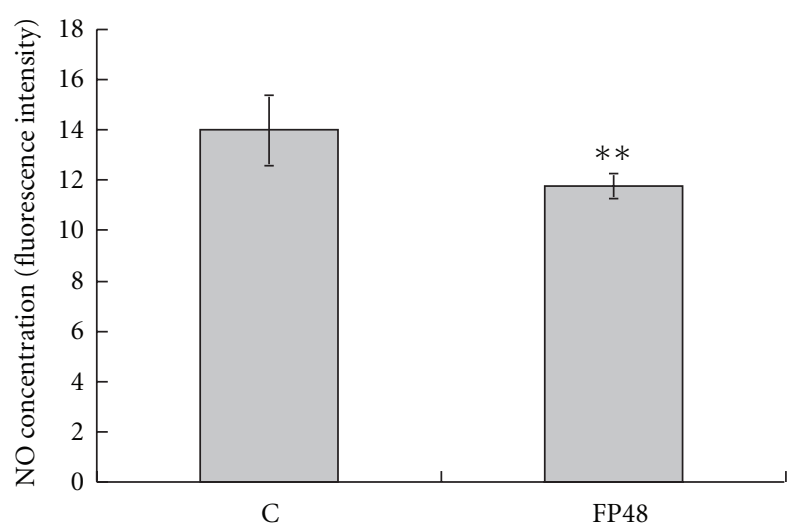

FIgURE 4: Comparison of intracellular NO between the control (group C) and cFIR (group FP48) groups. Expression values are the mean and standard deviation, and the statistical difference was detected by the Wilcoxon test.

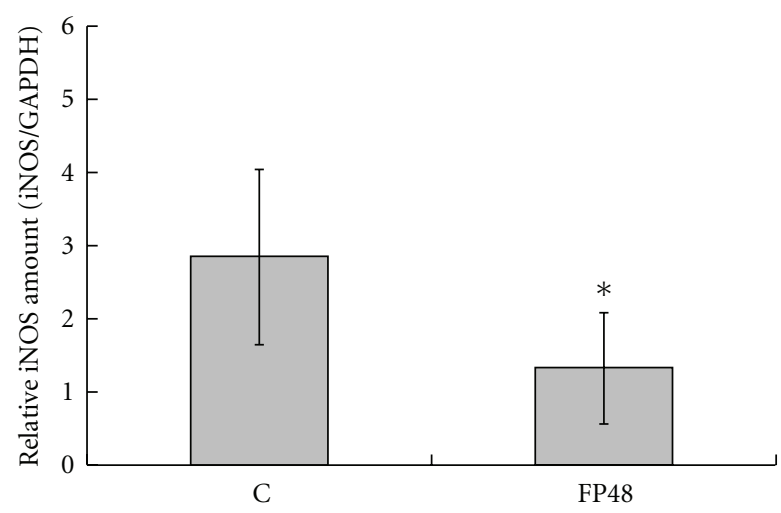

FIGURE 5: Comparison of inducible nitric oxide synthase between the control (group FP48) and cFIR (group FP48) groups. Expression values are the mean and standard deviation, and the difference between groups was tested using the Wilcoxon test.

growth of HeLa cells (a cervical cancer cell line). Similarly, Ishibashi et al.'s [6] demonstrated that hFIR suppressed the proliferation of several types of cancer cells, including HSC3 (tongue squamous cell carcinoma), Sa3 (gingival squamous cell carcinoma), and A549 (pulmonary adenocarcinoma) cell lines. Their results also demonstrated that hFIR has different effects on HSP70 overexpression in cancer cells with different basal levels of HSP70. Based on Ishibashi et al. [6] and our studies, FIR may have potential benefits in the medical treatment of melanomas. However, our study with cFIR differed from that of Ishibashi et al. with hFIR producing a $40^{\circ} \mathrm{C}$ thermal effect. As is known, HSPs accumulate in cells exposed to a heat source and a variety of other stressful stimuli. In fact, hFIR experiments produce a thermal effect which might overlap with the results of the somatothermal cFIR influence on cells.

Gene expression levels of HSPs can determine the fate of cells in response to a death stimulus, and apoptosisinhibitory HSPs, particularly HSP70, may participate in carcinogenesis [15]. A previous study demonstrated that pancreatic cancer cells expressed significantly higher HSP70 

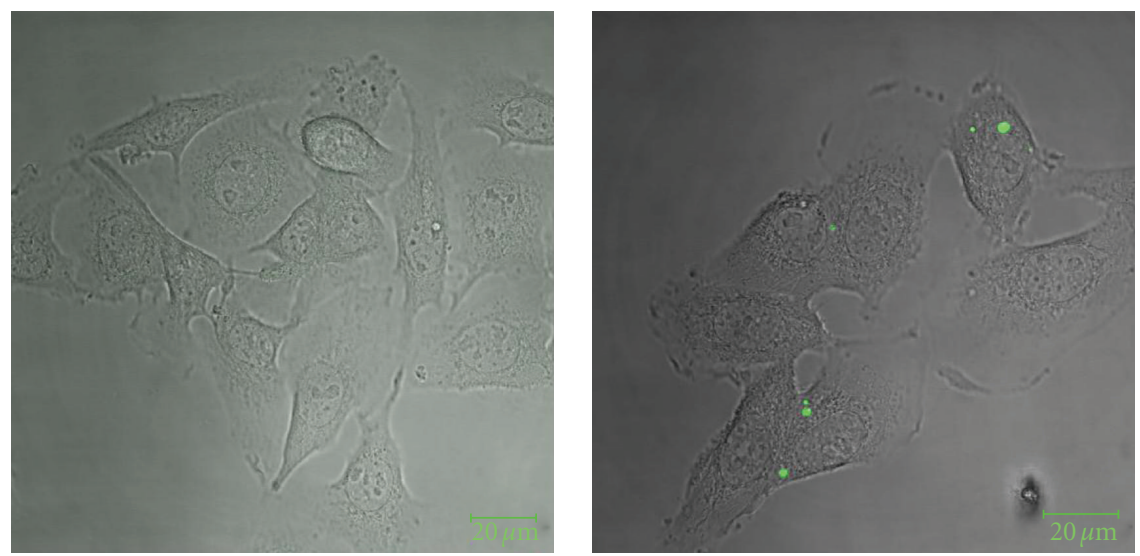

(a)

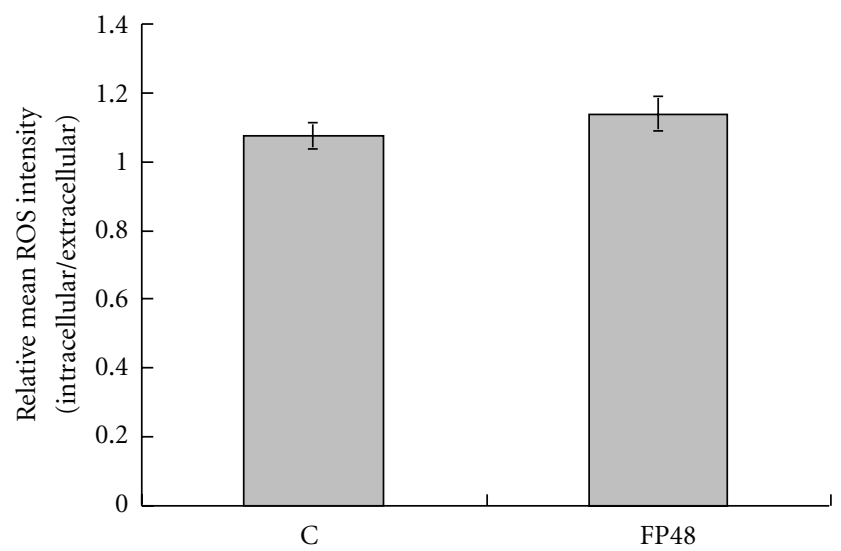

(b)

FIGURE 6: Intracellular ROS by confocal laser scanning microscopy. (a) Stained images of ROS in the control group (left) and cFIR group (right). Scale bars $=20 \mu \mathrm{m}$. (b) Comparison of normalized mean ROS fluorescence intensity in B16-F10 cells between the control (group C) and cFIR (group FP48) groups.

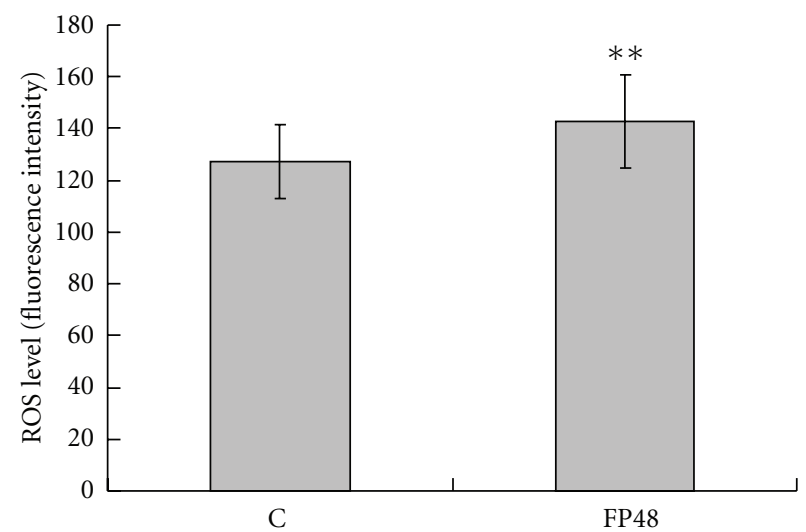

FIGURE 7: Intracellular ROS by flow cytometry. Expression values of the control (group C) and cFIR (group FP48) groups are the mean and standard deviation, and the statistical difference was detected by the Wilcoxon test.

levels compared to nonmalignant ductal cells, which suggests that HSP70 plays a role in tumor cell resistance to apoptosis [16]. They showed increased HSP70 expression in cancer tissues versus normal tissues from the same pancreatic cancer patient. These findings agree with several reports in the literature showing increased HSP70 expression in a variety of malignant tumors, such as colorectal, breast, and gastric cancers. The importance of these findings strengthens the hypothesis that high levels of HSP70 expression are correlated with increased drug resistance in cancer cell lines. They concluded that the major role of HSP70 was in boosting resistance of pancreatic cancer cells to apoptosis. Gurbuxani et al. [17] showed increased gene expression of HSP70 in tumor cells and proposed that it enhances their immunogenicity. However, HSP70 was also demonstrated to prevent tumor apoptosis. They proved that the reduced level of HSP70 expression in colon cancer cells resulted in a specific immune response by promoting cell death in vivo. HSP 70 is overexpressed in malignant melanomas [18] and underexpressed in renal cell cancer [19]. Overexpression of HSP70 in various tumors is associated with enhanced tumorigenicity and resistance to therapy. Conversely, downregulation of Hsp70 in tumor cells was found to enhance tumor regression in certain animal models [20].

HSP was found to be overexpressed by B16-F10 melanoma cells. The HSP70 protein content was shown to 


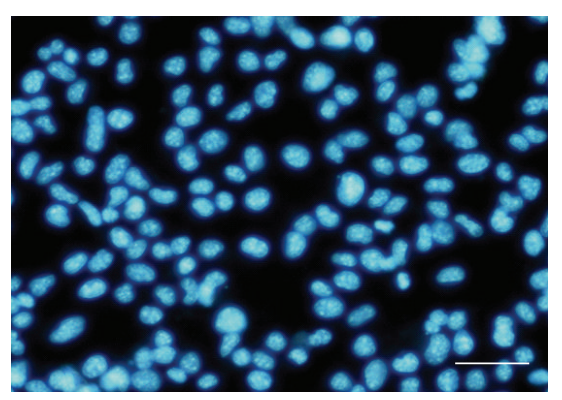

(a)

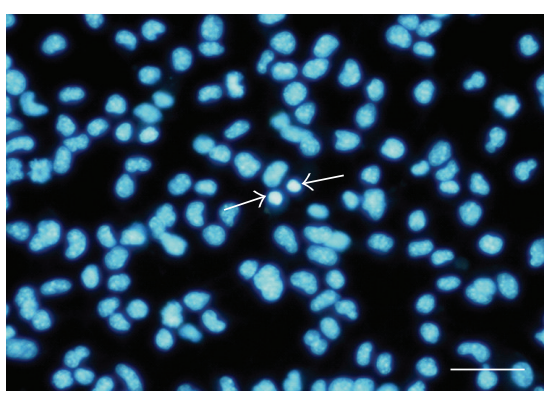

(b)

FIGURE 8: Hoechst 33342 staining to detect apoptotic changes. (a) Control group and (b) cFIR group. Scale bars $=50 \mu \mathrm{m}$. Arrows indicate very small amounts of apoptotic changes (DNA condensation) in B16-F10 cells treated by cFIR for $48 \mathrm{~h}$.

considerably vary in human melanoma cells from different cell lines, and HSP70 levels in melanoma cells evidently contribute to their resistance to anticancer drugs [21]. HSP70 expression is elevated in many cancers and contributes to tumor cell survival and resistance to therapy. Leu et al. [22] found that tumor cells cultured with an HSP70 inhibitor showed suppressed tumor development, and the survival of mice was enhanced. Stellas et al. [23] showed that using a monoclonal antibody against HSP90 was capable of inhibiting cell invasion and metastasis of B16F10 melanomas. Galluzzi et al. [24] also demonstrated that a chemical inhibitor of HSP70 exerted prominent tumorselective cytotoxic effects, thereby lending further support to the future application of HSP70 as a promising target for anticancer therapy. In addition, a previous study conducted by Nylandsted et al. [25] found that depletion of HSP70 may inhibit cancer. The expression of HSP70 is correlated with increased cell proliferation, poor differentiation, lymph node metastases, and poor therapeutic outcomes in human breast cancer [26]. A recent study validated that using an HSP70 inhibitor was capable of inhibiting B16-F10 cell growth [27].

In this study, we also found that NO levels significantly decreased after irradiation with cFIR powder, indicating that the inhibitory effect on the murine melanoma cell line may be associated with lowered levels of NO. Intracellular $\mathrm{NO}$ is a highly reactive molecule implicated in numerous physiologic and pathologic processes, which play important roles in nonspecific antitumor immune responses [8, 28, 29]. However, in some circumstances, NO may also lead to tumor expansion and metastasis $[28,30,31]$.

In this study, we further found that iNOS production in cFIR group was significantly lower than control group. This finding may reflect that the reduction of iNO is a result of the inhibition of iNOS by cFIR. For murine melanoma cells, a connection between elevated levels of NO after iNOS induction and consequent cancer cell inhibition was previously described [32]. In fact, iNOS was induced by cytokines and LPS in normal melanocytes but not in melanoma cells [33]. The expression of iNOS found in melanoma cells may result in the continuous formation of NO, which may subsequently activate or inhibit physiological processes different from apoptosis but important for tumor progression. The elevation of iNOS and the consequent higher NO levels were also associated with an increased number of lymphatic vessels, resulting in lymphangiogenesis in melanomas [34]. It was also demonstrated that the loss of iNOS inducibility in melanoma cells showed a well-demarcated difference from normal melanocytes, and this regulation defect was the result of melanocytic transformation and malignancy [31]. Unlimited elevation of $\mathrm{NO}$ concentrations in melanomas is expected to promote metastases by maintaining the vasodilator tone of blood vessels in and around the melanoma [35]. It is well recognized that NO is involved in melanoma progression, since the proliferative and metastatic capacities were measured and showed that NO-treated melanoma cells exhibited higher levels of aggressiveness [36]. Therefore, our results showing simultaneous melanoma cell inhibition and a decrease in NO by suppression of iNOS expression can logically be accepted.

ROS are constantly generated and eliminated in biological systems and play important roles in a variety of normal biochemical functions and abnormal pathological processes. Growing evidence suggests that cancer cells exhibit increased intrinsic ROS stress accompanied by increased metabolic activity and mitochondrial malfunction [37]. Previous studies demonstrated that certain agents that generate ROS help preferentially kill cancer cells or inhibit their growth [3841]. Cancer cells that exhibit increased intrinsic oxidative stress with high levels of cellular ROS and a low antioxidant capacity are more susceptible to chemotherapy. Therefore, there is a therapeutic strategy to treat cancer cells by further increasing ROS using pharmacological agents that directly increase ROS production, inhibit cancer cell antioxidant defenses, or their combination $[37,38]$.

Based on our current results, we propose that cFIR treatment may induce intracellular ROS production which results in cell growth arrest but not significant apoptosis [41]. On the other hand, cFIR treatment also reduced Hsp70 expression and NO production and resulted in further cell growth inhibition. However, the detailed mechanism is not clear yet, and further investigations are needed to elucidate this.

\section{Conclusions}

Unlike traditional FIR with a heat source, this study is the first to demonstrate that somatothermal cFIR without an 
additional thermal effect affected murine melanoma cells and was capable of suppressing the proliferation of B16-F10 cells and inhibiting intracellular NO and HSP70 production. Treatment with cFIR induced intracellular ROS production but did not significantly affect cell apoptosis, leading us to speculate that interference with the cell cycle, such as cell growth arrest, occurred. We deduced that the melanoma inhibitory effect may be a consequence of or share a common pathway with the decreased intracellular HSP70 and NO. Further investigations into the basic biomolecular and physiological mechanisms occurring in melanoma cells following cFIR treatment will help advance future therapeutic applications of cFIR.

\section{Acknowledgment}

This work was supported by a Grant from the National Science Council of Taiwan (NSC 99-2622-E-241-003-CC3).

\section{References}

[1] S. Inoue and M. Kabaya, "Biological activities caused by farinfrared radiation," International Journal of Biometeorology, vol. 33, no. 3, pp. 145-150, 1989.

[2] S. C. Lee, S. Y. Kim, S. M. Jeong, and J. H. Park, "Effect of farinfrared irradiation on catechins and nitrite scavenging activity of green tea," Journal of Agricultural and Food Chemistry, vol. 54, no. 2, pp. 399-403, 2006.

[3] S. Y. Yu, J. H. Chiu, S. D. Yang, Y. C. Hsu, W. Y. Lui, and C. $\mathrm{W}$. Wu, "Biological effect of far-infrared therapy on increasing skin microcirculation in rats," Photodermatology, Photoimmunology \& Photomedicine, vol. 22, no. 2, pp. 78-86, 2006.

[4] C. C. Lin, C. F. Chang, M. Y. Lai, T. W. Chen, P. C. Lee, and W. C. Yang, "Far-infrared therapy: a novel treatment to improve access blood flow and unassisted patency of arteriovenous fistula in hemodialysis patients," Journal of the American Society of Nephrology, vol. 18, no. 3, pp. 985-992, 2007.

[5] J. F. Tsai, S. Hsiao, and S. Y. Wang, "Infrared irradiation has potential antidepressant effect," Progress in Neuro-Psychopharmacology and Biological Psychiatry, vol. 31, no. 7, pp. 1397-1400, 2007.

[6] J. Ishibashi, K. Yamashita, T. Ishikawa et al., "The effects inhibiting the proliferation of cancer cells by far-infrared radiation (FIR) are controlled by the basal expression level of heat shock protein (HSP) 70A," Medical Oncology, vol. 25, no. 2, pp. 229-237, 2008.

[7] T. K. Leung, C. M. Lee, M. Y. Lin et al., "Far infrared ray irradiation induces intracellular generation of nitric oxide in breast cancer cells," Journal of Medical and Biological Engineering, vol. 29, no. 1, pp. 15-18, 2009.

[8] T. K. Leung, Y. S. Lin, Y. C. Chen et al., "Immunomodulatory effects of far-infrared ray irradiation via increasing calmodulin and nitric oxide production in raw 264.7 macrophages," Biomedical Engineering - Applications, Basis and Communications, vol. 21, no. 5, pp. 317-323, 2009.

[9] T. K. Leung, Y. S. Lin, C. M. Lee et al., "Direct and indirect effects of ceramic far infrared radiation on the hydrogen peroxide-scavenging capacity and on murine macrophages under oxidative stress," Journal of Medical and Biological Engineering. In press.

[10] A. H. Cory, T. C. Owen, J. A. Barltrop, and J. G. Cory, "Use of an aqueous soluble tetrazolium/formazan assay for cell growth assays in culture," Cancer Communications, vol. 3, no. 7, pp. 207-212, 1991.

[11] M. Sundaresan, Z. X. Yu, V. J. Ferrans, K. Irani, and T. Finkel, "Requirement for generation of $\mathrm{H} 2 \mathrm{O} 2$ for platelet-derived growth factor signal transduction," Science, vol. 270, no. 5234, pp. 296-299, 1995.

[12] J. A. Smith and M. J. Weidemann, "Further characterization of the neutrophil oxidative burst by flow cytometry," Journal of Immunological Methods, vol. 162, no. 2, pp. 261-268, 1993.

[13] S. Allen, J. Sotos, M. J. Sylte, and C. J. Czuprynski, "Use of hoechst 33342 staining to detect apoptotic changes in bovine mononuclear phagocytes infected with Mycobacterium avium subsp. paratuberculosis," Clinical and Diagnostic Laboratory Immunology, vol. 8, no. 2, pp. 460-464, 2001.

[14] F. Teraoka, Y. Hamada, and J. Takahashi, "Bamboo charcoal inhibits growth of HeLa cells in vitro," Dental Materials Journal, vol. 23, no. 4, pp. 633-637, 2004.

[15] C. Didelot, E. Schmitt, M. Brunet, L. Maingret, A. Parcellier, and C. Garrido, "Heat shock proteins: endogenous modulators of apoptotic cell death," Handbook of Experimental Pharmacology, vol. 172, pp. 171-198, 2006.

[16] A. Aghdassi, P. Phillips, V. Dudeja et al., "Heat shock protein 70 increases tumorigenicity and inhibits apoptosis in pancreatic adenocarcinoma," Cancer Research, vol. 67, no. 2, pp. 616625, 2007.

[17] S. Gurbuxani, J. M. Bruey, A. Fromentin et al., "Selective depletion of inducible HSP70 enhances immunogenicity of rat colon cancer cells," Oncogene, vol. 20, no. 51, pp. 7478-7485, 2001.

[18] N. Ricaniadis, A. Kataki, N. Agnantis, G. Androulakis, and C. P. Karakousis, "Long-term prognostic significance of HSP70, c-myc and HLA-DR expression in patients with malignant melanoma," European Journal of Surgical Oncology, vol. 27, no. 1, pp. 88-93, 2001.

[19] U. Ramp, C. Mahotka, S. Heikaus et al., "Expression of heat shock protein 70 in renal cell carcinoma and its relation to tumor progression and prognosis," Histology and Histopathology, vol. 22, no. 10, pp. 1099-1107, 2007.

[20] R. Dressel, C. Grzeszik, M. Kreiss et al., "Differential effect of acute and permanent heat shock protein 70 overexpression in tumor cells on lysability by cytotoxic T lymphocytes," Cancer Research, vol. 63, no. 23, pp. 8212-8220, 2003.

[21] N. V. Gukasova, I. G. Kondrasheva, E. Y. Moskaleva et al., "Hsp70 protein content in human melanoma cells as a factor of resistance to antitumor drugs," Molecular Medicine, vol. 1, pp. 15-21, 2009.

[22] J. I. J. Leu, J. Pimkina, A. Frank, M. E. Murphy, and D. L. George, "A small molecule inhibitor of inducible heat shock protein 70," Molecular Cell, vol. 36, no. 1, pp. 15-27, 2009.

[23] D. Stellas, A. Karameris, and E. Patsavoudi, "Monoclonal antibody 4C5 immunostains human melanomas and inhibits melanoma cell invasion and metastasis," Clinical Cancer Research, vol. 13, no. 6, pp. 1831-1838, 2007.

[24] L. Galluzzi, F. Giordanetto, and G. Kroemer, "Targeting HSP70 for cancer therapy," Molecular Cell, vol. 36, no. 2, pp. 176-177, 2009.

[25] J. Nylandsted, W. Wick, U. A. Hirt et al., "Eradication of glioblastoma, and breast and colon carcinoma xenografts by Hsp70 depletion," Cancer Research, vol. 62, no. 24, pp. 7139$7142,2002$.

[26] J. Nylandsted, M. Rohde, K. Brand, L. Bastholm, F. Elling, and M. Jäättelä, "Selective depletion of heat shock protein 70 (Hsp70) activates a tumor-specific death program that is independent of caspases and bypasses Bcl-2," Proceedings of the 
National Academy of Sciences of the United States of America, vol. 97, no. 14, pp. 7871-7876, 2000.

[27] A. Yerlikaya, E. Okur, S. Şeker, and N. Erin, "Combined effects of the proteasome inhibitor bortezomib and Hsp70 inhibitors on the B16F10 melanoma cell line," Molecular Medicine Reports, vol. 3, no. 2, pp. 333-339, 2010.

[28] J. M. Tarr, P. Eggleton, and P. G. Winyard, "Nitric oxide and the regulation of apoptosis in tumour cells," Current Pharmaceutical Design, vol. 12, no. 34, pp. 4445-4468, 2006.

[29] K. Ivanova, B. Lambers, R. Van Den Wijngaard et al., "Immortalization of human melanocytes does not alter the de novo properties of nitric oxide to induce cell detachment from extracellular matrix components via cGMP," In Vitro Cellular and Developmental Biology - Animal, vol. 44, no. 8-9, pp. 385395, 2008.

[30] B. Bonavida, S. Khineche, S. Huerta-Yepez, and H. Garbán, "Therapeutic potential of nitric oxide in cancer," Drug Resistance Updates, vol. 9, no. 3, pp. 157-173, 2006.

[31] R. Mitra, S. Singh, and A. Khar, "Antitumour immune responses," Expert Reviews in Molecular Medicine, vol. 5, no. 3, pp. 1-19, 2003.

[32] L. F. Fecker, J. Eberle, C. E. Orfanos, and C. C. Geilen, "Inducible nitric oxide synthase is expressed in normal human melanocytes but not in melanoma cells in response to tumor necrosis factor- $\alpha$, interferon- $\gamma$, and lipopolysaccharide," Journal of Investigative Dermatology, vol. 118, no. 6, pp. 1019-1025, 2002.

[33] L. C. Jadeski and P. K. Lala, "Nitric oxide synthase inhibition by $\mathrm{N}(\mathrm{G})$-nitro-L-arginine methyl ester inhibits tumor-induced angiogenesis in mammary tumors," American Journal of Pathology, vol. 155, no. 4, pp. 1381-1390, 1999.

[34] D. Massi, M. C. De Nisi, A. Franchi et al., "Inducible nitric oxide synthase expression in melanoma: Implications in lymphangiogenesis," Modern Pathology, vol. 22, no. 1, pp. 21-30, 2009.

[35] M. Joshi, J. Strandhoy, and W. L. White, "Nitric oxide synthase activity is up-regulated in melanoma cell lines: a potential mechanism for metastases formation," Melanoma Research, vol. 6, no. 2, pp. 121-126, 1996.

[36] Z. Yang, S. Yang, B. J. Misner, R. Chiu, F. Liu, and F. L. Meyskens, "Nitric oxide initiates progression of human melanoma via a feedback loop mediated by apurinic/apyrimidinic endonuclease-1/redox factor-1, which is inhibited by resveratrol," Molecular Cancer Therapeutics, vol. 7, no. 12, pp. 3751-3760, 2008.

[37] H. Pelicano, D. Carney, and P. Huang, "ROS stress in cancer cells and therapeutic implications," Drug Resistance Updates, vol. 7, no. 2, pp. 97-110, 2004.

[38] E. O. Hileman, J. Liu, M. Albitar, M. J. Keating, and P. Huang, "Intrinsic oxidative stress in cancer cells: a biochemical basis for therapeutic selectivity," Cancer Chemotherapy and Pharmacology, vol. 53, no. 3, pp. 209-219, 2004.

[39] H. Kadara, E. Tahara, H. J. Kim, D. Lotan, J. Myers, and R. Lotan, "Involvement of Rac in fenretinide-induced apoptosis," Cancer Research, vol. 68, no. 11, pp. 4416-4423, 2008.

[40] S. Macip, M. Igarashi, L. Fang et al., "Inhibition of p21-mediated ROS accumulation can rescue p21-induced senescence," EMBO Journal, vol. 21, no. 9, pp. 2180-2188, 2002.

[41] Y. F. Chang, Y. C. Hsu, H. F. Hung et al., "Quercetin induces oxidative stress and potentiates the apoptotic action of 2-methoxyestradiol in human hepatoma cells," Nutrition and Cancer, vol. 61, no. 5, pp. 735-745, 2009. 


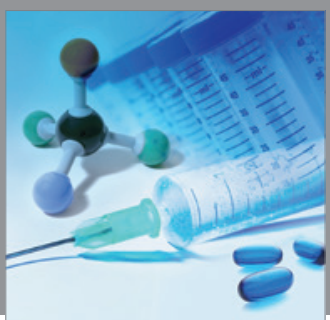

International Journal of

Medicinal Chemistry

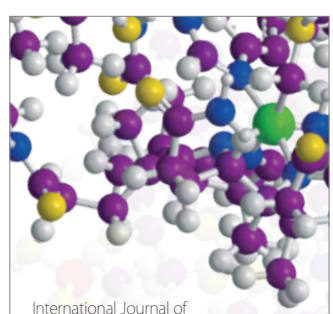

Carbohydrate Chemistry

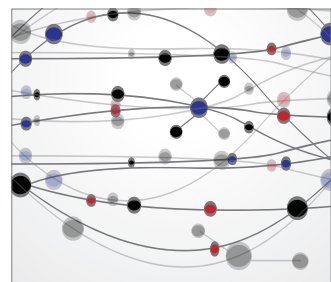

The Scientific World Journal
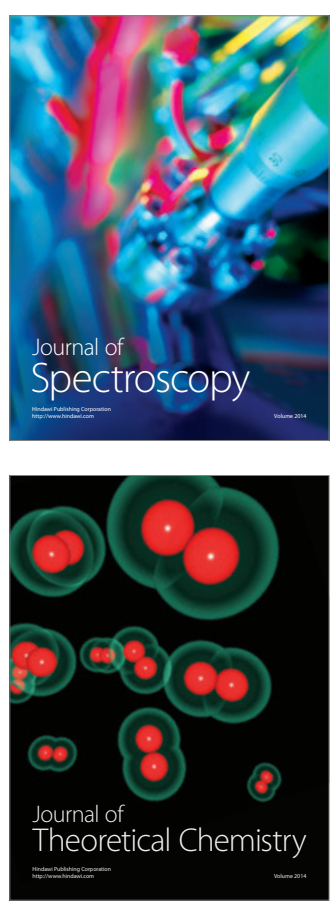
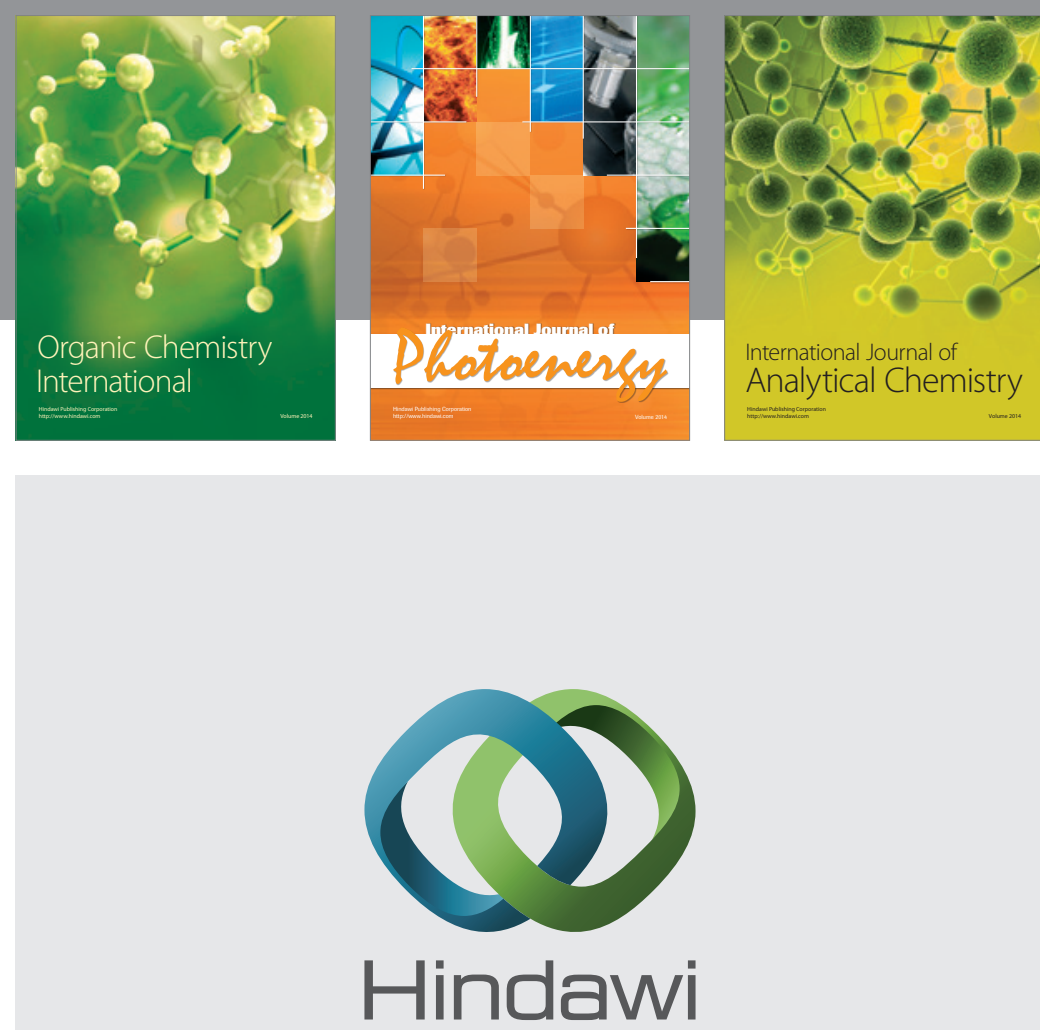

Submit your manuscripts at

http://www.hindawi.com
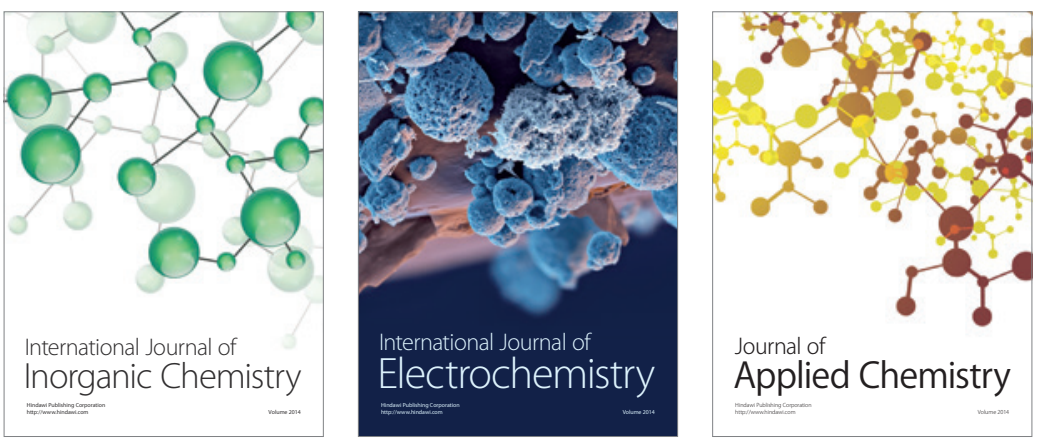

Journal of

Applied Chemistry
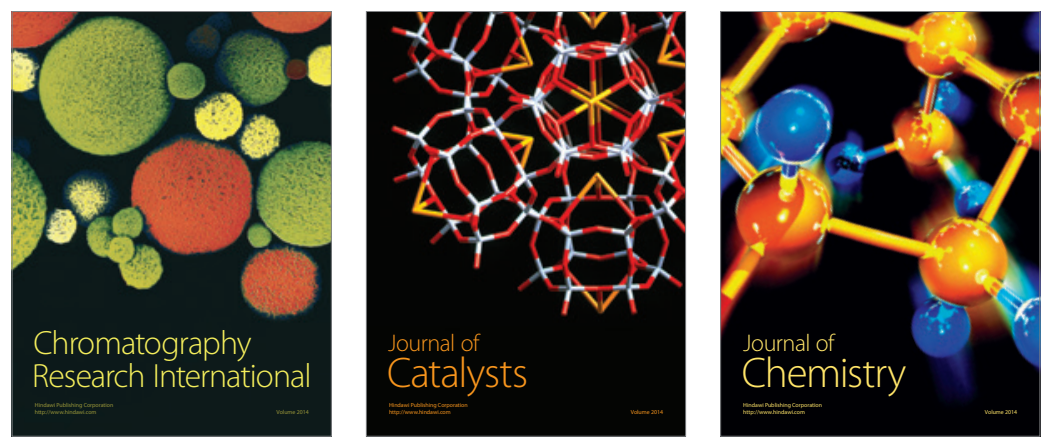
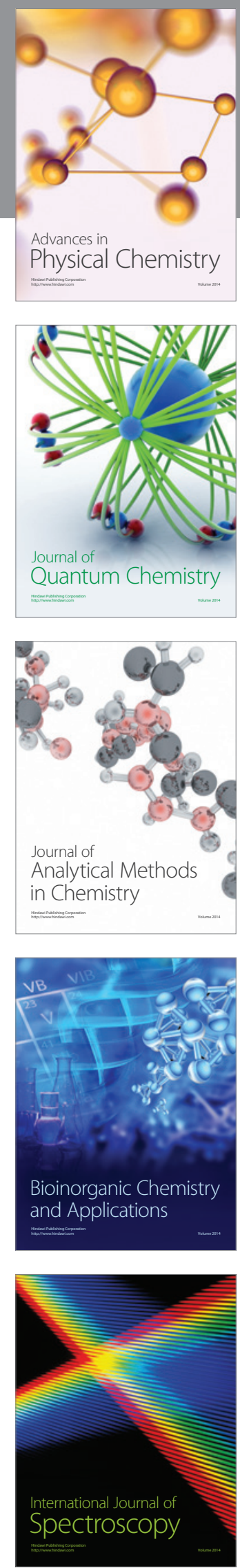